Pacific Journal of Mathematics

FINITE NETS. II. UNIQUENESS AND IMBEDDING 


\title{
FINITE NETS, II. UNIQUENESS AND IMBEDDING
}

\author{
R. H. BRUCK
}

1. Introduction. In discussing the present paper we have a choice of three languages: (a) the language of orthogonal latin squares; (b) the language of incomplete block designs, as used in connection with design of experiments; and (c) the geometric language of nets. As far as proofs are concerned, either (b) or (c) affords a useful symmetry which is missing in (a); it is merely a matter of taste that we choose (c). Here let us begin with (a).

Let $C$ be a collection of $t$ mutually orthogonal latin squares of side $n$. We assume $n>1, t \geqq 1$. The inequality $t \leqq n-1$ necessarily holds; if $t=n-1, C$ is said to be complete. As is well known, a complete set of orthogonal latin squares of side $n$ determines and is determined by an affine plane of order $n$. We define the degree, $k$, and deficiency, $d$, of $C$ by

$$
k=t+2, \quad d=n-1-t,
$$

so that

$$
k+d=n+1
$$

Here $k$ is, in language (b), the number of constraints: one constraint for the rows of the squares, one for the columns, and one for each of the $t$ squares. On the other hand, if $C$ can be enlarged to a complete set, $C^{\prime}$, of $n-1$ mutually orthogonal latin squares, then $d$ is the number of squares in $C^{\prime}$ which are not in $C$; or the number of constraints missing in $C$. In language (c) we may dessribe $C$ as a net $N$ of order $n$, degree $k$, deficiency $d$. For an example of such a net $N$, we may begin with an affine plane $\pi$ of order $n$-with its $n^{2}$ points and $n+1$ parallel classes of lines, $n$ lines per class-and retain the points but delete some $d$ parallel classes.

Before discussing the results of the paper, it will be convenient to define two polynomials $p(x), q(x)$ :

$$
\begin{aligned}
& p(x)=\frac{1}{2} x^{4}+x^{3}+x^{2}+\frac{3}{2} x, \\
& q(x)=2 x^{3}-x^{2}-x+1,
\end{aligned}
$$

Received November 22, 1961. Any views expressed in this paper are those of the author. They should not be interpreted as reflecting the views of The RAND Corporation or the official opinion or policy of any of its governmental or private research sponsors. Papers are reproduced by The RAND Corporation as a courtesy to members of its staff. 
and to note the following table:

$\begin{array}{rrrrrc}d & (d-1)^{2} & q(d-1) & 2(d-1)^{3} & p(d-1) & d^{4} / 2 \\ 1 & 0 & 1 & 0 & 0 & 1 / 2 \\ 2 & 1 & 1 & 2 & 4 & 8 \\ 3 & 4 & 11 & 16 & 23 & 401 / 2 \\ 4 & 9 & 43 & 54 & 81 & 128 \\ 5 & 16 & 109 & 128 & 214 & 3121 / 2 \\ 6 & 25 & 221 & 250 & 470 & 648\end{array}$

We note from (1.1) that the side $n$ and deficiency $d$ of the collection $C$ (of mutually orthogonal latin squares) satisfy the inequality $n \geqq$ $d+2$. Assuming that $d \geqq 1$, we are interested in conditions under which $C$ can be completed; that is, can be enlarged to a complete set, $C^{\prime}$, of mutually orthogonal latin squares of side $n$. Our first result is:

(A) If $n>(d-1)^{2}$, and if $C$ can be completed at all, then it can be completed uniquely, aside from trivialities.

This follows from Theorem 3.1. However, examples show that the condition $n>(d-1)^{2}$ does not ensure completion. On the other hand:

(B) If $n>p(d-1), C$ can always be completed.

This follows from Theorem 4.3. The result (B) is known to be best possible for $d=1$ (folk-lore) and for $d=2$ (Shrikhande [9]). Whether (B) is best possible for $d>2$ is unknown to the author. Before mentioning further results, intermediate between (A) and (B), which take into account the structure of $C$, it seems worthwhile to give a simple consequence of (B).

Bose and Shrikhande defined $m(n)$ to be the maximum number of mutually orthogonal latin squares of side $n$. As a result of the work of Bose, Shrikhande and Parker (see, for example, [1]), Chowla, Erdos and Straus [5] were able to prove that

$$
m(n)>\frac{1}{3} n^{1 / 91}
$$

for all sufficiently large $n$ (the lower bound on $n$ being unknown.) In view of $(B)$ we may state a dichotomy:

Either (I) $m(n)=n-1$

or (II) $n \leqq p(n-2-m(n))<\frac{1}{2}[n-1-m(n)]^{4}$.

As an easily stated consequence:

$$
\text { If } m(n)<n-1 \text {, then } m(n)<n-1-(2 n)^{1 / 4} \text {. }
$$


We note that (I) holds precisely when there exists an affine or projective plane of order $n$. Thus (I) holds for infinitely many $n$, for example, for every prime-power. However, by the Bruck-Ryser Theorem (Bruck and Ryser [3]), (II) also holds for infinitely many $n$. We may add that, just as Chowla et al. state that their methods would not allow (1.5) to be improved to

$$
m(n)>n^{1 / 2}
$$

(although (1.7) is not known to be false for large $n$ ), so it seems likely that the present methods would not allow (1.6) to be improved to

$$
\text { If } m(n)<n-1 \text {, then } m(n) \leqq n-2-n^{1 / 2} .
$$

Note that (1.8) would result from (II) if we could replace $p(x)$ by $x^{2}$. -A more reasonable possibility is that $p(x)$ could be replaced by $q(x)$, by dint of a more penetrating discussion of maximal incomplete sets of orthogonal latin squares. This would give an exponent $1 / 3$, instead of $1 / 2$, in (1.8).--But even if (1.7), (1.8) could both be proved, they would still leave a great gap in our knowledge of $m(n)$.

The refinements of (A), (B) are conveniently stated in terms of graphs. From the collection $C$-or, equivalently, from the corresponding net $N$ of order $n$, degree $k$, deficiency $d$-we define a graph $G_{1}$ with $n^{2}$ vertices, whose edges are the unordered pairs of distinct points lying on a common line of the net $N$. If $G_{2}$ is the complementary graph of $G_{1}$ then $G_{2}$ has (at least superficially) the type of structure that one would associate with the graph of a net of order $n$, degree $d$, deficiency $k$. (Note that the roles of $k$ and $d$ have been interchanged.) We abstract from this superficial structure a definition of what we call a pseudo net-graph of order $n$, degree $d$, deficiency $k$. Our first observation is that, to enlarge $C$ to a complete set $C^{\prime}$, or, equivalently, to imbed the net $N$ in an affine plane of order $n$, we must introduce a suitable collection of lines into the complimentary graph $G_{2}$ in such a way as to turn $G_{2}$ into the graph of a net of order $n$, degree $d$, deficiency $k$. We actually prove our results for pseudo net-graphs. Thus (B) is obtained as a consequence of:

(B') If $n>p(d-1)$, every pseudo net-graph of order $n$, degree $d$ is the graph of a uniquely defined net of order $n$, degree $d$.

The corresponding theorem for $d=2$ was proved by Shrikhande [10], who also refers to unpublished results of Dale Mesner for $d \geqq 2$. In the language (b) used by Shrikhande, $\left(\mathrm{B}^{\prime}\right)$ could be restated as:

$\left(\mathrm{B}^{\prime \prime}\right)$ If $n>p(d-1)$, and if the parameters of the second kind 
for a partially balanced incomplete block design with $n^{2}$ treatments with two associate classes are given by

$$
n_{1}=d(n-1), \quad p_{11}^{1}=n-2+(d-1)(d-2), \quad p_{11}^{2}=d(d-1),
$$

then the design has $L_{d}$ association scheme.

Now we require the notion of a claw. If $G$ is a pseudo net-graph, a claw $P, S$ of $G$ is a pair consisting of a vertex $P$ and a nonempty set $S$ of vertices distinct from $P$ such that $P$ is joined in $G$ to every vertex in $S$ but no two vertices in $S$ are joined in $G$. The order of the claw is the cardinal number, $|S|$, of $S$. If $G$ is the graph of a net of degree $d$ then, obviously, $G$ has no claws of order $d+1$. We may state a partial converse (see Theorem 4.2):

(C) If $n>2(d-1)^{3}$, and if $G$ is a pseudo net-graph of order $n$, degree $d$ which possesses no claws of order $d+1$, then $G$ is the graph of a uniquely defined net of order $n$, degree $d$.

This result is also given by Shrikhande [10] for $d=2$. We may remark here that the inequality in $(\mathrm{C})$ could probably be sharpened to

$$
n>2(d-1)^{3}-(d-1)^{2} .
$$

This could be done if the right-hand side of formula (4.7) in Lemma 4.2 could be replaced by $d-1$, as seems likely.

To state our final result in this direction we need the notion of a grand clique. A clique (of a pseudo-net graph $G$ of order $n$, degree $d$ ) is a set of vertices every two of which are joined in $G$. And a grand clique is a maximal clique containing at least

$$
n-(d-1)^{2}(d-2)
$$

vertices. Our result is (Theorem 4.1):

(D) Assume $n>q(d-1)$, and let $G$ be a pseudo net-graph of order $n$, degree $d$ such that $(i)$ no two distinct grand cliques of $G$ have more than one common vertex and (ii) $G$ has no claws of order $d+1$. Then $G$ is the graph of a uniquely defined ne s of order $n$, degree $d$.

We may remark that, for $d=1$ or 2 , condition (i) of (D) may be dropped. Indeed, in these cases, grand cliques have exactly $n$ vertices, and this simplifies matters considerably. On the other hand, for $d>2$, (i) is needed to help us prove that grand cliques have exactly $n$ vertices and are in fact the lines of a net. 
These are perhaps the main results of the paper. However, other items are also worthy of note. In $\S 2$ we find it worthwhile to formalize the familiar process of "enumeratinng in two ways." We feel that this process would repay formal study, just as the formal study of equality has led to a rich theory of equivalence relations. In $\S 5$ (originally conceived as a section designed to end all study of incidence matrices, but now recast) we uncover a one-to-one correspondence, apparently unknown until the present, between sets of $k-2$ mutually orthogonal latin squares of side $n$ and sets of $k$ mutually orthogonal matrices of order $n^{2}$. (Theorem 5.1). The suitable modification for pseudo net-graphs is given in Theorem 5.2. We also show in $\S 5$ that a conjecture concerning adjacency matrices of finite graphs (originally advanced by Harary and disproved by Bose) is hopelessly beyond repair.

The paper [4], of like title to the present one, was compressed at the suggestion of the editors. A good deal of material-some of which appears in almost unrecognizable form in $\S \S 3,5$ of the present paperwas omitted, including all examples. There are some grounds for our belief that the result was to hamper theory of latin squares. As a case in point, a counterexample contained in the original version of [4], and known to the author in 1949, served in 1961 to halt an extensive high-speed machine program on latin squares. With this in mind, we have tried in the concluding section $(\S 6)$ to include a reasonable selection of remarks and examples.

In conclusion, the author would like to express his appreciation to The RAND Corporation of Santa Monica and to all the participants of the 1961 Summer Symposium on Combinatorial Mathematics of Project RAND. The present paper has been largely molded in discussions with Alan Hoffman, R. C. Bose and E. T. Parker. Hoffman is certainly the father of Lemma 4.4 (though he is not responsible for (4.19)), and Hoffman and Bose must share some guilt in connection with the birth of Theorem 5.1-which they, however, have never seen.

2. Counting in two ways. During the course of this paper we shall have many occasions to use the familiar process of "counting in two ways." In order to ensure brevity without loss of clarity, it seems worthwhile to state the process as a formal lemma. Here, for any set $S,|S|$ denotes the cardinal number of $S$.

Lemma 2.1. Let $A, B$ be nonempty sets, $\rho$ be a finite subset of the direct product set $A \times B$. For each $a$ in $A$, let a $\rho$ denote the subset of $B$ consisting of all $b$ in $B$ such that $(a, b)$ is in $\rho$; and, for each $b$ in $B$, let $\rho b$ be the subset of $A$ consisting of all $a$ in $A$ such 
that $(a, b)$ is in $\rho$. Then

$$
\sum_{a \in A}|a \rho|=\sum_{b \in B}|\rho b| .
$$

Proof. For each $a$ in $A$, the set $(a, a \rho)$, consisting of all pairs $(a, b)$ with $b$ in $a \rho$, contains precisely $|a \rho|$ elements of $\rho$. Also, the sets $(a, a \rho)$, as $a$ ranges over $A$, partition $\rho$-provided we ignore the empty sets which may turn up. Hence the left-hand side of (2.1) is equal to $|\rho|$. Similarly for the right-hand side of (2.1). This completes the proof.

It goes without saying that the value of (2.1) in any particular case depends upon skill in choosing the sets $A$ and $B$ (these may often be complex sets constructed from others more immediately at hand) and the relation (or finite subset) $\rho$. I would conjecture that all proofs by enumeration may be reduced to a sequence of applications of the apparently innocuous Lemma 2.1. Be that as it may, there were several instances at the 1961 Combinatorial Symposium of Project RAND in which Lemma 2.1 provided a simpler alternative to proofs involving matrix calculations.

3. Nets. We begin with a positive integer (or, more generally, with any cardinal number) $k$ such that

$$
k \geqq 3 \text {. }
$$

A $k$-net, $N$, is a system of undefined points and lines, together with an incidence relation, subject to the following axioms: (i) $N$ has at least one point. (ii) The lines of $N$ are partitioned into $k$ disjoint, nonempty, "parallel classes" such that (a) each point of $N$ is incident with exactly one line of each class; (b) to two lines belonging to distinct classes there corresponds exactly one point of $N$ which is incident with both lines. For convenience, we shall use phrases such as "point is on line" instead of speaking of incidence.

The axioms, coupled with (3.1), ensure the existence of two distinct lines $L, L^{\prime}$ of $N$ and a parallel class $K$ containing neither of $L, L^{\prime}$. Since each point of $L$ lies on a unique line of class $K$, and since each line of class $K$ meets $L$ in a unique point, there is a one-to-one correspondence between the points of $L$ and the lines of $K$. Similarly, there is a one-to-one correspondence between the points of $L^{\prime}$ and the lines of $K$. Furthermore, each point of $N$ lies on exactly one line of $K$. Hence, if some line of $N$ contains exactly $n$ distinct points, the following statements are true: $n \geqq 1$.

( I ) Each line of $N$ contains exactly $n$ distinct points, where 
(II) Each point of $N$ lies on exactly $k$ distinct lines, where $\pi \geqq 1$.

(III) $N$ has exactly $k n$ distinct lines. These fall into $k$ parallel classes of $n$ lines each. Distinct lines of the same parallel class have no common points. Two lines of different classes have exactly one common point.

(IV) $N$ has exactly $n^{2}$ distinct points.

A system $N$ satisfying (I)-(IV) we shall call a net of order $n$, degree k. If (3.1) fails-in particular, if $k=1$ or 2 -we shall call the net degenerate. And if $n=1$ we shall call the net trivial. In the sequel we study finite nontrivial nets ( $n$ and $k$ finite) but we cannot entirely avoid degenerate nets.

For each finite nontrivial net $N$ of order $n$, degree $k$ we introduce integers $d, n_{i}$ and $p_{j k}^{i}$ as follows:

$$
\begin{array}{rr} 
& k+d=n+1 \\
n_{1}=k(n-1), & n_{2}=d(n-1), \\
& p_{11}^{1}=n-2+(k-1)(k-2), \\
p_{12}^{1}= & p_{21}^{1}= \\
p_{22}^{1}= & (k-1) d, \\
p_{22}^{2}=n-2+(d-1)(d-2), \\
p_{21}^{2}=p_{12}^{2}= & (d-1) k, \\
p_{11}^{2}= & k(k-1) .
\end{array}
$$

In (3.3), (3.4) we are using the notation of R. C. Bose [2]. We call the integer $d$ the deficiency of $N$. It is to be observed that interchange of $k$ and $d$ preserves (3.2) and has the effect in (3.3), (3.4) of interchanging the subscripts and superscripts 1,2 .

Before making clear the significance of the above definitions, it will be convenient to introduce further notation. If $P, Q$ are two distinct points of $N$ we say that $P, Q$ are joined in $N$ if there exists a line $P Q$ of $N$ (necessarily unique) which contains both $P$ and $Q$; if the line $P Q$ does not exist, we say that $P, Q$ are not joined in $N$. By a partial transversal, $S$, of $N$ we mean a nonempty set, $S$, of points of $N$ such that every two distinct points in $S$ are not joined in $N$. By a transversal of $N$ we mean a partial transversal with exactly $n$ distinct points (where $n$ is the order of $N$ ). We are now ready for an important elementary lemma.

LEMMA 3.1. Let $N$ be a nontrivial finite net of order $n$, degree k, deficiency $d$.

(i) If $S$ is a partial transversal of $N$, then $|S| \leqq n$. 
(ii) If $P$ is a point of $N$, then, of the $n^{2}-1$ points of $N$ distinct from $P, n_{1}$ are joined to $P$ in $N$ and $n_{2}$ are not joined to $P$ in $N$.

(iii) If $P$ is a point of $N$ and if $L$ is a line of $N$ not containing $P$, then $P$ is joined to $k-1$ points of $L$ and $P$ is not joined to $d$ points of $L$.

(iii') If $P$ is a point of $N$ and if $T$ is a transversal of $N$ not containing $P$, then $P$ is not joined to $d-1$ points of $T$ and $P$ is joined to $k$ points of $T$.

(iv) If $P, Q$ are distinct points joined in $N$, then, of the remaining $n^{2}-2$ points, $p_{11}^{1}$ are joined to both of $P, Q ; p_{12}^{1}$ are joined to $P$ and not joined to $Q ; p_{21}^{1}$ are not joined to $P$ and joined to $Q ; p_{22}^{1}$ are not joined to $P$ and not joined to $Q$.

(iv') If $P, Q$ are distinct points not joined in $N$, then, of the remaining $n^{2}-2$ points, $p_{22}^{2}$ are not joined to $P$ and not joined to $Q ; p_{21}^{2}$ are not joined to $P$ and joined to $Q ; p_{12}^{2}$ are joined to $P$ and not joined to $Q ; p_{11}^{2}$ are joined to $P$ and joined to $Q$.

REMARKs. (1) The statement of Lemma 3.1 is intended to emphasize a duality of importance for the sequel. Item (i) merely points out that transversals are maximal partial transversals. (However, not every maximal partial transversal is a transversal.) We note that if "joined" and "not joined" are interchanged, then (ii) remains true provided $n_{1}, n_{2}$ are interchanged; (iii) and (iii') are interchanged provided "line $L$ " and "transversal $T$ " are interchanged, as well as $k$ and $d$; and (iv), (iv') are interchanged provided the subscripts and superscripts 1, 2 are interchanged.

(2) In view of (ii)-since $n_{2}=d(n-1)$-or (iii) we see that the deficiency, $d$, of a finite net $N$, is a nonnegative integer.

(3) In view of (ii) we see that a finite net, $N$, of order $n$, deficiency zero is precisely an affine plane of order $n$. Thus the deficiency measures the extent to which a net fails to be an affine plane-namely, it lacks $d$ classes of parallel lines.

(4) In view of (iii') we see that if $d=0$, then $N$ has no transversals. (Indeed, if $d=0$, each partial transversal of $N$ has exactly one point, since every two distinct points are joined in $N$-by (ii), (3.3).)

Proof (i). Let $s=|S|$ and let $K$ be any parallel class of lines of $N$. Each of the $s$ points of $S$ lies on a unique line of $K$. Two distinct points of $S$ are not joined in $N$ and hence lie on distinct lines 
of $K$. Therefore $s \leqq|K|=n$. This proves (i).

(ii) We note from (3.3), (3.2) that $n_{1}+n_{2}=n^{2}-1$. Each of the $k$ lines through $P$ contains $n-1$ points in addition to $P$. The $n_{1}=k(n-1)$ points so obtained are distinct and are all the points joined to $P$. This proves (ii).

(iii) We note from (3.2) that $(k-1)+d=n$. One of the $k$ lines through $P$ is parallel to $L$. The rest meet $L$ in $k-1$ distinct points. Moreover, $L$ has exactly $n$ distinct points. This proves (iii).

(iii') The $n$ distinct points of $T$ lie one each on the $n$ distinct lines of each parallel class (cf. the proof of (i)). Hence the $k$ lines through $P$ meet $T$ in $k$ distinct points. Since $k+(d-1)=n$, there remain $d-1$ points of $T$ not joined to $P$. This proves (iii').

(iv) Here $P, Q$ lie on a line $P Q$ of $N$. There are $n-2$ points of $P Q$ which are joined to both $P$ and $Q$. Each of the $k-1$ lines through $P$, other than $P Q$, is met by the $k-1$ lines through $Q$, other than $P Q$, in $k-2$ distinct points (there being a case of parallelism). This gives a total of

$$
n-2+(k-1)(k-2)=p_{11}^{1}
$$

distinct points joined to both $P$ and $Q$. Since

$$
p_{11}^{1}+p_{12}^{1}=n_{1}-1
$$

by (3.4), (3.2), (3.3), and since $P$ is joined (by (ii)) to exactly $n_{1}-1$ points distinct from itself and $Q$, then there are exactly $p_{12}^{1}$ distinct points joined to $P$ but not to $Q$. (And, of course, there are $p_{21}^{1}$ distinct points joined to $Q$ but not to $P$.) Since

$$
p_{21}^{1}+p_{22}^{1}=n_{2}
$$

by $\left(3.4,(3.2),(3.3)\right.$, and since $P$ is not joined (by (ii)) to exactly $n_{2}$ distinct points, then there are exactly $p_{22}^{1}$ distinct points joined to neither $P$ nor $Q$. This proves (iv).

(iv') Here $P, Q$ are not joined in $N$. Since each of the $k$ lines through $P$ is met by the $k$ lines through $Q$ in exactly $k-1$ points (one case of parallelism) and since none of these intersection points is $P$ or $Q$, there are exactly

$$
k(k-1)=p_{11}^{2}
$$

distinct points joined to both $P$ and $Q$. Since

$$
p_{11}^{2}+p_{12}^{2}=n_{1} \text {, }
$$

there are exactly $p_{12}^{2}$ points joined to $P$ but not $Q$. Since

$$
p_{21}^{2}+p_{22}^{2}=n_{2}-1
$$


and since $P$ is not joined to exactly $n_{2}-1$ points in addition to $Q$, the proof of (iv') and of Lemma 3.1 is now complete.

It will be convenient at this point to make a brief review of some well known facts about nets. Let $n \geqq 2$ be any given positive integer, and let us construct a square of side $n$, containing $n^{2}$ cells. We regard the cells as points of a net $N$. If we define two distinct cells to be joined in $N$ if and only if they lie in the same row, then $N$ is a. (degenerate) net of order $n$, degree 1 , with the $n$ rows of cells as the $n$ lines of its single parallel class. If we allow both rows and columns. of cells as lines, we have a (degenerate) net of order $n$, degree 2. If we now mark the cells with the numbers 1 through $n$ in such a way as to form a latin square and allow, in addition to the row-lines and column-lines, lines consisting of $n$ cells marked with the same number, we get a net of order $n$, degree 3. Similarly, for any integer $k$ in the range $3 \leqq k \leqq n+1$, a set of $k-2$ mutually orthogonal latin squares of side $n$ may be used to define a (non-degenerate) net. of order $n$, degree $k$. Conversely, any net of order $n$, degree $k(k \geqq 1)$. can be obtained in the manner indicated, usually in many ways.

To imbed a net $N$ of order $n$, degree $k$ (where $k<n+1$ ) in a net $N_{1}$ of order $n$, degree $k+1$ which has the same points as $N$ and has $k$ of its line classes identical with those of $N$ is equivalent to finding a single new "parallel class." This must consist of $n$ distinct transversals of $N$, no two with a point in common. To imbed $N$ in an affine plane $N_{2}$ of order $n$ (with the same points as $N$ and with $k$ of its line classes identical with those of $N$ ) is equivalent to finding $d=n+1-k$ new "parallel classes," consisting of $d$ sets of $n$ parallel transversals, such that two distinct transversals belonging to the same set have no common point and two belonging to different sets have exactly one common point. It is easy to see that each of the $n^{2}$ points should lie in exactly $d$ of the transversals. Indeed, to imbed net $N$ of order $n$, degree $k$, deficiency $d>0$ in an affine plane is equivalent to defining a complementary net, $N^{\prime}$, of order $n$, degree $d$, deficiency $k$, whose points are identical with those of $N$ and whose lines are a. suitably selected set of transversals of $N$.

Several problems arise. A given net may have no complementary net or several complementary nets. How can we ensure existence or uniqueness of a complementary net? A given net may have several classes of parallel transversals, or no complete parallel class of transversals, or no transversals at all. How can we ensure existence of a. suitable collection of transversals?

One case in which transversals are embarrassingly common is worth mentioning. A net of order 10 , degree 3 , is essentially a latin square of side 10 . Here the deficiency is $d=8$. To imbed such a 
net in an affine plane of order 10 we would need a suitable collection of $d n=80$ transversals, 8 through each point. No such collection has ever been found. However, E. T. Parker, in a machine search for an orthogonal mate to suitably selected latin squares, usually finds an average of about 120 transversals per cell-or about 15 times as many transverals per point of the net as we would want. As we shall see, the situation changes when the order $n$ is somewhat "larger" compared with the deficiency $d$.

LEMma 3.2. Let $N$ be a finite nontrivial net of order $n$, degree $k$, deficiency $d>0$. Let $T$ be a transversal of $N$ and let $S$ be a partial transversal of $N$ not contained in $T$ but containing at least two points of $T$. Then

$$
\begin{aligned}
|S \cap T| & \leqq d-1, \\
|S| & \leqq(d-1)^{2} .
\end{aligned}
$$

CoRollary. If $N$ is a finite nontrivial net of order $n$, deficiency $d>0$, and if $n>(d-1)^{2}$, then two distinct transversals of $N$ can have at most one common point.

Proof. By hypothesis, $S$ contains at least one point $R$ which is not in $T$. By Lemma 1 (iii'), there are precisely $d-1$ points of $T$ not joined to $R$. Among these $d-1$ points must be the points of $S \cap T$, since $R$ is joined to no other point of $S$. Hence we have (3.5). Again, by hypothesis, $S \cap T$ contains at least two distinct points $P, Q$. By Lemma 1 (iv'), there are precisely $p_{22}^{2}$ points joined to neither $P$ nor $Q$, and the points of $S \cup T-\{P, Q\}$ must be among these $p_{22}^{2}$ points. Hence

$$
|S \cup T| \leqq p_{22}^{2}+2=n+(d-1)(d-2) .
$$

By this and (3.5), we have

$$
\begin{aligned}
|S|+|T| & =|S \cap T|+|S \cup T| \\
& \leqq(d-1)+n+(d-1)(d-2)=n+(d-1)^{2} .
\end{aligned}
$$

However, $|T|=n$, since $T$ is a transversal. Therefore we have (3.6). If we assume that $S$ is also a transversal, (3.6) yields

$$
n \leqq(d-1)^{2} \text {. }
$$

At this point we note that if $S, T$ are any two distinct transversals, then $S$ must have a point not in $T$. Hence, if we further assume that $S, T$ have at least two common points, we get (3.8). Thus, by denying (3.8), we get the Corollary. This completes the proof. 
Two remarks are in order. First, if (3.8) holds, then (3.6) is trivial in view of Lemma 1 (i). Secondly, the Corollory to Lemma 3.2 is "best possible" of its kind. One class of examples may be obtained as follows: Let $m$ be any integer (for example, any prime power) for which there exists an affine plane $\pi$ of order $n=m^{2}$ which possesses an affine subplane $\pi_{1}$ of order $m$. We form a net $N$ of order $n=m^{2}$, degree $k=m^{2}-m$, deficiency $d=m+1$ whose points are the points of $\pi$ and whose lines are the $k$ parallel classes of $\pi$ containing no lines of $\pi_{1}$. The net is degenerate if $m=2$, and nondegenerate otherwise. Among the transversals of $N$ are the $m^{2}+m$ lines of $\pi_{1}$ (that is, the lines of $\pi$ containing at least two and hence exactly $m$ points of $\pi_{1}$ ) and each two of these intersect in at most one point. But there is another transversal, namely the set consisting of the $n=m^{2}$ points of $\pi_{1}$, and this has exactly $m$ points in common with each of the lines of $\pi_{1}$. In this class of examples we have $n=(d-1)^{2}$. In addition, when equality holds in (3.8), transversals seem to behave as the above discussion indicates. Indeed:

LEMma 3.3. Let $N$ be a finite net of order $n=m^{2}$, degree $k=$ $m^{2}-m$, deficiency $d=m+1$. Assume $m>2$, so that $N$ is nontrivial and nondegenerate.

(i) If $S, T$ are distinct transversals with more than one common point, then they have exactly $d-1=m$ common points. Moreover (a) each point of $S-T$ is joined to each point of $T-S$ and (b) if $P, Q$ are any two distinct points of the intersection $S \cap T$, then every point not in the union $S \cup T$ is joined to at least one of $P, Q$.

(ii) If $S, T, U$ are three distinct transversals such that $S$ has $m$ points in common with each of $T, U$, then $T, U$ have at most one common point.

Proof. For (i), we use the proof of Lemma 3.2, assuming that $S, T$ are distinct transversals with at least two common points. Then (3.5), (3.7) become

$$
|S \cap T| \leqq m, \quad|S \cup T| \leqq 2+p_{22}^{2}=m^{2}+m(m-1),
$$

and we get

$$
2 n=|S|+|T|=|S \cup T|+|S \cap T| \leqq 2 m^{2}=2 n .
$$

Hence we must have equality in (3.9). Thus $|S \cap T|=m$, and, moreover, (b) holds. Again, if $R$ is any point in $S-T$, then $R$ is not joined to exactly $d-1=m$ points of $T$, and these points must be the points of $S \cap T$. Consequently, $R$ must be joined to every point of $T-S$. This proves (a) and completes the proof of (i). 
To prove (ii), we begin by assuming that $S \cap T \cap U$ has at least two distinct points $P, Q$. Then, by (i) (b), since $S \cap T$ has $m$ points and since no point of $U$ is joined to $P$ or $Q$, we must conclude that $U$ is in $S \cup T$. Since, by (i) (a), every point of $S-T$ is joined to every point of $T-S$, we see that $U$ cannot contain both a point of $S-T$ and a point of $T-S$. Therefore either $U \subset S$ or $U \subset T$. But then, since $|U|=|S|=|T|$, either $U=S$ or $U=T$, in contradiction to hypothesis. Consequently,

$$
|S \cap T \cap U| \leqq 1 .
$$

By hypothesis, $|S \cap T|=m=|S \cap U|$. By (3.10), $S \cap T, S \cap U$ have at most one common point. These two facts, taken together, tell us that $U$ has at least one point of $S-T$ (indeed, at least $m-1$ such points). Therefore $U$, having a point of $S-T$, can have no point of $T-S$. This means that

$$
T \cap U \subset S \cap T \cap U .
$$

And (13.10a), (3.10) complete the proof of Lemma 3.3.

There are many other examples indicating that the Corollary to Lemma 3.2 is best possible. One comes from the nets of order $n=6$, degree $k=3$, deficiency $d=4$. Here we have $n=6<9=(d-1)^{2}$. Such nets are given by latin squares of order 6 . There are 17 types, and at least one has two distinct transversals with 3 common points. (See Fisher and Yates [6].)

As far as construction is concerned, the nets satisfying (3.8) are the most important at present. Nevertheless, there is a great deal to be learned about the remaining nets, and we shall be concerned here with inequalities at least as strong as

$$
n>(d-1)^{2} \text {. }
$$

The most obvious consequences of $\left(3.11^{*}\right)$ are summed up in the following theorem.

TheOREM 3.1. Let $N$ be a finite nontrivial net of order $n$, degree $k$, deficiency $d$, satisfying (3.11*). Let $N^{*}$ be the system whose points are the points of $N$ and whose lines are the lines of $N$ together with the transversals of $N$, and whose incidence relation is the natural one.

(i) If $t$ is the total number of distinct transversals of $N$, then

$$
t \leqq d n
$$

(ii) A necessary and sufficient condition that $N$ be imbeddable in an affine plane of order $n$ is that equality hold in (3.12).

(iii) If $N$ is imbeddable in an affine plane $N_{1}$ of order $n$, then 
$N_{1}$ is isomorphic to $N^{*}$. In summary, $N^{*}$ is the only candidate for an affine plane of order $n$ containing $N$.

Proof. By the Corollary to Lemma 3.2, two distinct transversals. of $N$ have at most one common point. Moreover, two distinct lines of $N$ have at most one common point, and a line and a transversal of $N$ have exactly one common point. Consequently, two distinct lines: of $N^{*}$ have at most one common point.

For each point $P$ of $N$ (and $N^{*}$ ), let $t(P)$ be the number of distinct. transversals of $N$ containing $P$. Thus the number of distinct lines of $N^{*}$ containing $P$ is exactly

$$
k+t(P) .
$$

Two such lines have only the point $P$ in common. Therefore the number of points, distinct from $P$, to which $P$ can be joined in $N^{*}$ (not $N$ !) is

$$
[k+t(P)](n-1) \leqq n^{2}-1 .
$$

Since $n>1$, we deduce that

$$
k+t(P) \leqq n+1=k+d
$$

and hence that

$$
t(P) \leqq d
$$

for every point $P$ in $N$. Moreover, for any fixed $P$, equality holds in (3.13) precisely when $P$ can be joined (in $N^{*}$ ) to every other point. By summing (3.13) over the $n^{2}$ points $P$ of $N$, and remembering that every transversal has exactly $n$ points, we see that (3.12) holds, with equality precisely when every two distinct points are joined in $N^{*}$. In particular, (i) is true.

If $N$ is imbeddable in an affine plane $N_{1}$ of order $n$, then (when $N$ is considered as a subsystem of $N_{1}$ ) every line of $N_{1}$ is either a line of $N$ or a transversal of $N$. Hence every line of $N_{1}$ is a line of $N^{*}$. Since every two distinct points are joined in $N_{1}$, we must conclude that equality holds in (3.12).

Now suppose, conversely, that equality holds in (3.12). Then, also, equality holds in (3.13) for every point $P$, and every two distinct. points are joined in $N^{*}$. We consider a transversal $T$ and a line $L$ of $N$ and note the $T, L$ have a unique common point, $Q$. Let $P$ be any point of $L$ distinct from $Q$. Then $P$ is not in $T$. Hence, by Lemma 1 (iii'), there are axactly $d-1$ distinct points of $T$ not joined in $N$ to $P$. Each of these is joined to $P$ by a unique line of $N^{*}$, giving, in all, $d-1$ distinct transversals of $N$ which contain $P$ and 
intersect $T$. Since $t(P)=d$, there remains a unique transversal which contains $P$ and if parallel to $T$. As $P$ varies over the $n-1$ points of $L$ distinct from $Q$, we get in this way $n-1$ distinct transversals parallel to $T$. No two of these transversals intersect, for a common point $R$ would lie on two distinct transversals parallel to $T$. Consequently, when we include $T$, we get a set of $n$ distinct, mutually parallel transversals. These must contain all the points of $N$, namely $n$ points on each of $n$ transversals. It should now be clear that the $t=d n$ transversals of $N$ form $d$ distinct parallel classes of lines of $N^{*}$, distinct from the $k n$ lines of $N$. Therefore $N^{*}$ is a net of order $n$, degree $k+d=n+1$, deficiency 0 . That is, $N^{*}$ is affine plane.

Putting the last two paragraphs together, we see that (ii) and (iii) are true. This completes the proof of Theorem 3.1.

It would be wrong to assume that the $N^{*}$ of Theorem 3.1 is always an affine plane. If $N$ is the net of order $n=4$, degree $k=3$, deficiency $d=2$ given by the cyclic group of order 4 then (3.11) holds but $N$ has no transversals. If $N$ is the net of order $n=5$, degree $k=3$, deficiency $d=3$ given by any loop of order 5 other than the cyclic group (there are only two nets of order 5, degree 3) then $\left(3.11^{*}\right)$ holds but $N$ has exactly 3 transversals; one point lies on all three, 12 points lie each on one, and 12 points lie on none. Moreover (see Norton [8]) there exists a net of order $n=7$, degree $k=5$, deficiency $d=3$ with too few transversals to be imbedded in a net of degree 6 , deficiency 2. Precise necessary and sufficient conditions, in the presence of $\left(3.11^{*}\right)$, that $N^{*}$ be an affine plane still await exploration.

In the section which follows we show, in particular, that a suitable strengthening of the inequality $\left(3.11^{*}\right)$ suffices to ensure that $N^{*}$ is an affine plane.

4. Net-graphs and pseudo net-graphs. From a net $N$ of order $n$, degree $k$, deficiency $d$ we form a net-graph $G_{1}$ of order $n$, degree $k$, deficiency $d$ (namely, the graph of $N$ ) as follows: $G_{1}$ has $n^{2}$ vertices, namely the $n^{2}$ points of $N$. Two distinct points $P, Q$ of $N$ form an (unordered) edge $\{P, Q\}$ of $G_{1}$ if and only if $P, Q$ are joined in $N$ (that is, lie on a common line of $N$.) Since the edges of $G_{1}$ are unordered, $G_{1}$ is a symmetric graph. Since each vertex of $G_{1}$ lies on exactly $n_{1}=k(n-1)$ edges of $G_{1}$, the graph is regular. But $G_{1}$ has still more regularity, given in Lemma 3.1 (iv) and (iv') in terms of the constants of connection $p_{j k}^{i}$.

For any symmetric graph $G$, the complementary graph $G^{\prime}$ is a symmetric graph with the same vertices as $G$, such that, if $P, Q$ are distinct vertices of $G$, then $\{P, Q\}$ is on edge of $G^{\prime}$ precisely when $\{P, Q\}$ is not an edge of $G$.

In particular, if $G_{1}$ is as in the first paragraph, and if $G_{2}$ is the 
complement of $G_{1}$, then $G_{2}$ is an example of what we shall call a pseudo net-graph of order $n$, degree $d$, deficiency $k$. And the question as to whether $N$ can be imbedded (in at least one way) in an affine plane of order $n$ is (as essentially noted in $\S 3$ ) equivalent to the question as to whether $G_{2}$ is the net-graph of at least one net $N^{\prime}$ of order $n$, degree $d$, deficiency $k$, namely a net complementary to $N$. Moreover, by Theorem 3.1, if $n>(d-1)^{2}$, and if $G_{2}$ is a net-graph, then the corresponding net is uniquely defined by $G_{2}$.

By a pseudo net-graph $G$ of order $n$, degree $d$, deficiency $k$, where $n, d, k$ are nonnegative integers related by

$$
d+k=n+1,
$$

we mean a symmetric graph with $n^{2}$ vertices such that

(i) each vertex of $G$ is joined (by an edge of $G$ ) to exactly

$$
n_{1}=d(n-1)
$$

other vertices of $G$;

(ii) two distinct vertices $P, Q$ of $G$ which are joined in $G$ are together joined to exactly

$$
p_{11}^{1}=n-2+(d-1)(d-2)
$$

other vertices of $G$;

(iii) two distinct vertices $P, Q$ which are not joined in $G$ are together joined to exactly

$$
p_{11}^{2}=d(d-1)
$$

other vertices in $G$.

It will be noted that we have interchanged $k$ and $d$ and the indices 1 and 2 in formulas (3.2), (3.3), (3.4). This is merely a matter of convenience in view of the application to imbedding of nets. We shall have little need to refer to the deficiency, $k$, of $G$. However, to avoid trivialities, we shall assume throughout that

$$
n \geqq d \geqq 1 \text {. }
$$

By a clique of graph $G$ we mean a subgraph of $G$ every two of whose vertices are joined in the subgraph. That is, a clique is a complete subgraph of $G$. We are interested in introducing certain cliques as lines. Specifically, if $G$ is a pseudo net-graph of order $n$, we define a line of $G$ to be a clique with exactly $n$ vertices. When $G$ is the complementary graph, $G_{2}$, of a net $N$, the cliques of $G$ are the partial transversals of $N$, and the lines of $G$ are the transversals of $N$. In this case, by Lemma 3.1 (i), no clique of $G$ has more than $n$ elements. The same fact is true for pseudo net-graphs, but requires 
a different proof.

Lemma 4.1. Let $G$ be a pseudo net-graph of order $n$, degree $d$, and let $L$ be a line of $G$. Then

(i) each vertex of $G$ which is not in $L$ is joined in $G$ to exactly $d-1$ distinct vertices of $L$; and

(ii) $L$ is a maximal clique of $G$.

CoRollary. No clique of $G$ has more than $n$ elements.

Proof. Let $L^{\prime}$ be the set consisting of the $n^{2}-n$ vertices of $G$ which are not in $L$. For each integer $x$ in the range $0 \leqq x \leqq n$, let $g(x)$ denote the number of vertices in $L^{\prime}$ which are joined in $G$ to exactly $x$ distinct vertices in $L$. We shall first make use of the formulas

$$
\begin{aligned}
\sum g(x) & =n^{2}-n, \\
\sum x g(x) & =(d-1)\left(n^{2}-n\right), \\
\sum x^{2} g(x) & =(d-1)^{2}\left(n^{2}-n\right),
\end{aligned}
$$

where the sum in each case is over the range of $x$, and then establish them later. From these formulas we deduce that

$$
\begin{aligned}
& \sum(d-1-x)^{2} g(x) \\
& \quad=\left(n^{2}-n\right)\left[(d-1)^{2} \cdot 1-2(d-1) \cdot(d-1)+1 \cdot(d-1)^{2}\right]=0,
\end{aligned}
$$

and thence that $g(x)=0$ for $x \neq d-1$. At this point, (4.3) yields $g(d-1)=n^{2}-n$. And now (i) follows. From (i) and the fact that $n$ exceeds $d-1$, we see that for every vertex $P$ in $L^{\prime}$, the set $L \cup\{P\}$ is not a clique, since $P$ is joined to only $d-1$ vertices, and therefore is not joined to all vertices, in $L$. This means that $L$ is a maximal clique.-The Corollary should be obvious.

We prove the formulas by appeal to Lemma 2.1. In each case, the set $B$ of that lemma is $L^{\prime}$. For (4.3), $A$ is any one-element set, and $\rho$ is $A \times B$. For (4.4), $A$ is $L$ and $\rho$ is the set of all pairs $(a, b)$ with $a$ in $A, b$ in $B$ such that $\{a, b\}$ is an edge. The left side of (4.4) is a double sum; $x g(x)$ counts all $|\rho b|$ with $b$ joined to exactly $x$ edges, and $\sum x g(x)$ gives the complete sum. For the right-hand side, we note that there are $n$ choices of $a$ in $A$. Each $a$ lies on $d(n-1)$ edges, including $n-1$ edges joining it to points of $A=L$. Hence

$$
\sum|\alpha \rho|=n \cdot[d(n-1)-(n-1)]=(d-1)\left(n^{2}-n\right) .
$$

To get (4.5), we take $A$ to be the set of $n(n-1)$ ordered pairs of distinct vertices of $L$, and $\rho$ to be the subset of $A \times B$ consisting of 
all pairs $(a, b)$ such that $b$ is joined by an edge to both of the vertices making up $a$. Then $x(x-1) g(x)$ is the sum of $|\rho b|$ over all $b$ which are joined to exactly $x$ vertices of $L$, and

$$
\sum x(x-1) g(x)=\sum|\rho b| \text {. }
$$

On the other hand, for each element $a$ of $A$,

$$
|a \rho|=p_{11}^{1}-(n-2)=(d-1)(d-2) .
$$

Thus

$$
\sum x(x-1) g(x)=\left(n^{2}-n\right)(d-1)(d-2),
$$

whence, by addition of (4.4), we get (4.5). This completes the proof of Lemma 4.1. It seems worth remarking that, although Lemma 4.1 and its proof both seem pretty obvious, the proof was still lacking for several weeks after everything which follows in this section had been established subject to the conjecture that no clique had more than $n$ vertices.

In the proofs which follow, we first establish the existence of certain cliques called grand cliques, and eventually prove, on the basis of Lemma 4.1, that these are lines. We make two definitions, relative to a pseudo net-graph of order $n$, degree $k$ :

A major clique, $K$, is a clique such that

$$
|K| \geqq n-(d-1)^{2}(d-2) \text {. }
$$

A grand clique is a major clique which is also a maximal clique. We note from Lemma 4.1 that, if $d=1$ or 2, major cliques and grand cliques are the same as lines. There is a lemma for graphs completely analogous to Lemma 3.2 (with lines and cliques replacing transversals and partial transversals) but here we need something weaker:

Lemma 4.2. Let $G$ be a pseudo net-graph of order $n$, degree $d$, and let $K, L$ be two distinct cliques of $G$.

(i) If $K \cup L$ is not a clique, then

$$
|K \cap L| \leqq d(d-1) .
$$

(ii) If $K \cap L$ has at least two vertices, then

$$
|K \cup L| \leqq n+(d-1)(d-2) \text {. }
$$

(iii) If (4.7), (4.8) hold, then

$$
|K|+|L| \leqq n+2(d-1)^{2} .
$$

Corollary. If $G$ is a pseudo net-graph of order $n$, degree $d$, 
and if $n>2(d-1)^{3}$, then two distinct grand cliques of $G$ can have at most one common vertex.

REMARK. Analogous results hold for partial transversals in a net of order $n$, deficiency $d$.

Proof. (i) If $K \cup L$ is not a clique, there must exist a vertex $P$ in $K-L$ and a vertex $Q$ in $L-K$ such that $P, Q$ are not joined in $G$. Then $P, Q$ are together joined to exactly

$$
p_{11}^{2}=d(d-1)
$$

other vertices, and these must include $K \cap L$. This proves (i).

(ii) If $K \cap L$ contains two distinct vertices $R, S$, then $R, S$ are are joined in $G$ and hence are together joined to exactly

$$
p_{11}^{1}=n-2+(d-1)(d-2)
$$

other vertices. Among these must be included $K \cup L-\{R, S\}$. This proves (ii); and (iii) follows immediately.

Now suppose that $K, L$ are two distinct maximal cliques with at least two common vertices. Then (ii) holds. Moreover, $K \cup L$ cannot be a clique, so (i) holds. Therefore we have (iii). If $K, L$ are also both major cliques, (4.9) yields

$$
2\left[n-(d-1)^{2}(d-2)\right] \leqq n+2(d-1)^{2}
$$

and hence

$$
n \leqq 2(d-1)^{3}
$$

Consequently, two distinct grand cliques cannot have two common vertices unless (4.10) holds. This proves the Corollary.

To establish the existence of major and grand cliques, we need the concept of a claw-a concept suggested in conversation by Alan Hoffman. By a claw, $P, S$, of a pseudo net-graph, $G$ is meant an ordered pair consisting of a vertex $P$, the vertex of the claw, and a nonempty set $S$ of vertices distinct from $P$ such that every vertex in $S$ is joined to $P$ in $G$ but no two vertices in $S$ are joined in $G$. By the order of the claw $P, S$ we mean the number, $|S|$, of vertices in $S$.

When $G$ is the complementary graph of a net $N$ of deficiency $d$, it is easy to see that a claw $P, S$ of order $d$ exists for every vertex $P$. Indeed, let $L$ be any line of $N$ not containing $P$, and let $S$ consist of the $d$ distinct points of $L$ not joined to $P$ in $N$; then every two points of $S$ are joined in $N$. Hence, in $G, P, S$ is a claw of order $d$ with vertex $P$.

We need several lemmas concerning claws, and it is convenient 
to begin with a fairly general preliminary lemma.

Lemma 4.3. Let $G$ be a pseudo net-graph of order $n$, degree d, and let $P, S$ be a claw of $G$ of order $|S|=s$. Let $T$ be the set of all vertices of $G$ other than $P$ and those in $S$. For each $x$ in the range $0 \leqq x \leqq s$, let $f(x)$ be the number of vertices in $T$ which are joined to $P$ and, in addition, are joined to exactly $x$ vertices in $S$. Then

$$
\begin{gathered}
\sum_{0}^{s} f(x)=d(n-1)-s \\
f(0)-\sum_{2}^{s}(x-1) f(x)=(d-s)(n-1)-s(d-1)(d-2) \\
2 f(0)+\sum_{3}^{s}(x-1)(x-2) f(x) \\
=\alpha_{s}+2(d-s)(n-1)-2 s(d-1)(d-2)
\end{gathered}
$$

where $\alpha_{s}$ is an integer such that

$$
0 \leqq \alpha_{s} \leqq s(s-1)\left(d^{2}-d-1\right),
$$

and the upper bound is attained in (4.14) precisely when every vertex of $T$ which is joined to at least two distinct vertices of $S$ is also joined to $P$.

REMARK. If $s \leqq 2$, the summation on the left side of (4.13) should be omitted. Similarly, if $s=1$, the summation on the left side of (4.12) should be omitted.

Proof. The left-hand side of (4.11) is the number of vertices of $T$ which are joined to $P$. As for the right-hand side of (4.11), $P$ is joined in $G$ to exactly $d(n-1)$ distinct vertices; of these vertices, $s$ are in $S$ and the rest are in $T$. This proves (4.11).

Next we prove

$$
\sum_{1}^{s} x f(x)=s[n-2+(d-1)(d-2)],
$$

by applying Lemma 2.1. We take $A$ to be the set of all vertices in $T$ which are joined to $P, B$ to be $S$, and $\rho$ to be the subset of $A \times B$ consisting of all $(a, b), a \in A, b \in B$, such that $\{a, b\}$ is an edge of $G$. For any $x \geqq 1, x f(x)$ is the sum of $|a \rho|$ as $a$ ranges over the vertices in $A$ which are joined to exactly $x$ vertices in $B=S$; hence the lefthand side of (4.15) is $|\rho|=\sum|a \rho|$. For any $b$ in $B=S$, since $P$ and $b$ are joined, there are exactly $p_{11}^{1}$ vertices in $G$ joined to both $P$ and $b$; and these are in $A$. Hence 


$$
|\rho b|=p_{11}^{1}=n-2+(d-1)(d-2)
$$

and therefore, since $|B|=|S|=s, \Sigma|\rho b|$ is the right-hand side of (4.15).

This proves (4.15). To get (4.12), we subtract (4.15) from (4.11). Next we prove

$$
\sum_{2}^{s} x(x-1) f(x)=\alpha_{s}
$$

where $\alpha_{s}$ satisfies (4.14). To do this we first define, for every ordered pair $U, V$ of distinct vertices in $S, f_{1}(U, V)$ to be the number of vertices in $T$ which are joined to $U, V$ and also to $P$, and $f_{0}(U, V)$ to be the number of vertices in $T$ which are joined to $U, V$ but not to $P$. For each such pair $U, V$, there are exactly $p_{11}^{2}$ vertices in $G$ joined to both of $U, V$; one of these vertices is $P$ and the rest are in $T$. Hence

$$
f_{1}(U, V)+f_{0}(U, V)=p_{11}^{2}-1=d^{2}-d-1 .
$$

We define

$$
\alpha_{s}=\sum f_{1}(U, V)
$$

where the sum is over the $s(s-1)$ ordered pairs of vertices $U, V$ in $S$, and observe that

$$
\alpha_{s}+\sum f_{0}(U, V)=s(s-1)\left(d^{2}-d-1\right) .
$$

Since the second sum is a nonnegative integer, we see that the integer $\alpha_{s}$ satisfies (4.14) and attains its upper bound under the conditions stated in the lemma. To prove (4.16) we use Lemma 2.1 with $A$ as before and with $B$ defined to be the set of all ordered pairs $U, V$ of distinct vertices in $S$. Also, $\rho$ is the subset of $A \times B$ consisting of all triples $(a, U, V)$ with $a$ joined to both of $U, V$. From the definition of $\alpha_{s},|\rho|=\sum|\rho b|=\alpha_{s}$. For each $x \geqq 2, x(x-1) f(x)$ is the sum of $|a \rho|$ over all $a$ in $A$ which are joined to exactly $x$ elements of $S$. Thus we have (4.16). To obtain (4.13), we multiply (4.12) by 2 and add the result to (4.16). This completes the proof of Lemma 4.3.

The author is indebted to Allan Hoffman for suggesting the importance of the non-existence of claws of order $d+1$, and for sketching a non-existence proof for $n$ large compared with $d$. In the next lemma we give precise details in terms of the polynomial $p(x)$ defined by

$$
p(x)=\frac{1}{2} x^{4}+x^{3}+x^{2}+\frac{3}{2} x .
$$

It will be convenient to note that 


$$
2[p(d-1)-1]=(d+1) d\left(d^{2}-d-1\right)-2(d+1)(d-1)(d-2) .
$$

Lemma 4.4. If $G$ is a pseudo net-graph of order $n$, degree $d$, and if

$$
n>p(d-1),
$$

then $G$ has no claws of order $d+1$.

Proof. Assume, by way of obtaining a contradiction, that $G$ has a claw $P, S$ of order $d+1$. Then we may quote Lemma 4.3 with $s=d+1$. The left hand side of (4.13) is a nonnegative integer. Hence, certainly, if we replace $\alpha_{d+1}$ by its upper bound in (4.14), the right hand side of (4.13) must be nonnegative. This gives

$$
(d+1) d\left(d^{2}-d-1\right)-2(n-1)-2(d+1)(d-1)(d-2) \geqq 0
$$

and hence, by (4.18),

$$
2(n-1) \leqq 2[p(d-1)-1],
$$

in contradiction to (4.19). This proves Lemma 4.4.

The next three lemmas may conveniently be stated and proved together:

LEMma 4.5. Let $G$ be a pseudo net-graph of order $n$, degree $d$ such that

$$
n-1>(d-1)^{2}(d-2) \text {. }
$$

Then to every pair $P, Q$ of distinct vertices joined in $G$ there corresponds at least one claw $P, S$ of order $d$ such that $S$ contains $Q$.

Lemma 4.6. Let $G$ be a pseudo net-graph of order $n$, degree $d$ such that (4.20) holds and $G$ has no claws of order $d+1$. Then every edge of $G$ is contained in at least one grand clique of $G$.

LEMmA 4.7. Let $G$ be a pseudo net-graph of order $n$, degree $d$ subject to the following three conditions: (i) $G$ has no claws of order $d+1$; (ii) two distinct grand cliques of $G$ have at most one common point and (iii) $n>q(d-1)$ where

$$
q(x)=2 x^{3}-x^{2}-x+1 .
$$

Then every vertex of $G$ lies in exactly $d$ distinct grand cliques, and every grand clique of $G$ is a line of $G$.

Proof of Lemma 4.5. We begin by noting that $P,\{Q\}$ is a claw 
of order one. If $d=1$, the proof of Lemma 4.5 is complete. Therefore we consider the case $d>1$ and assume inductively that there exists a claw $P, S$ of order $s$ such that $S$ contains $Q$ and $1 \leqq s \leqq$ $d-1$. Since $s \leqq d-1$, the right-hand side of (4.12) (see Lemma 4.3) is at least

$$
n-1-(d-1)^{2}(d-2) .
$$

Since the sum on the left-hand side of (4.12) is non-negative, we deduce that

$$
f(0) \geqq n-1-(d-1)^{2}(d-2)>0,
$$

the last inequality following from (4.20). If $R$ is any one of the $f(0)$ vertices in $T$ which are joined to $P$ but to no vertex in $S$, then $P$, $S \cup\{R\}$ is a claw of order $s+1$. Therefore, by mathematical induction, we have the conclusion of Lemma 4.5.

Proof of Lemma 4.6. Let $\{P, Q\}$ be any edge of $G$. By Lemma 4.5, there exists at least one claw $P, S^{\prime}$ of order $d$ such that $S^{\prime}$ contains $Q$. We write $S^{\prime}=\{Q\} \cup S$ where $S$ does not contain $Q$. Then (in the notation of Lemma 4.3) let $H$ be the set of all elements of $T$ which are joined to $P$ but to no element of $S$. Clearly $H$ contains $Q$. Moreover, $|H|=f(0)$, and $f(0)$ satisfies (4.22). Hence if $K=\{P\} \cup H$,

$$
|K| \geqq n-(d-1)^{2}(d-2) \text {. }
$$

We claim that $K$ is a clique. Indeed, every element of $H$ is joined to $P$. Therefore, if $K$ contains two distinct vertices $A, B$ not joined in $G$, then $P, S \cup\{A, B\}$ is a claw of order $d+1$, contrary to hypothesis. In view of (4.23), the clique $K$ is major. Therefore, if $K^{\prime}$ is any maximal clique containing $K$, then $K^{\prime}$ is a grand clique containing the edge $\{P, Q\}$. This completes the proof of Lemma 4.6.

Proof of Lemma 4.7. We first note that

$$
q(d-1)-1=(d-1)^{2}(d-2)+d(d-1)(d-2) .
$$

Hence the inequality $n>q(d-1)$ implies the inequality (4.20). If $P$ is any vertex of $G$, there exists, by Lemma 4.5 , at least one claw $P, S$ of order $d$ with vertex $P$. We denote the $d$ vertices in $S$ by $A_{1}, A_{2}, \cdots, A_{d}$. For each $i$ in the range $1 \leqq i \leqq d$, we denote by $H_{i}$ the set of vertices, distinct from $P$ and the $A_{j}$ for $j \neq i$; which are joined to $P$ but to no vertex $A_{j}$ for $j \neq i$. As the proof of Lemma 4.6 shows, $P \cup H_{i}$ is, for each $i$, a major clique containing $P$ and $A_{i}$. We denote by $K_{i}$ a grand clique containing $P \cup H_{i}$. Since, for $i \neq j$, $H_{i}$ and $H_{j}$ have no common elements, it follows from our uniqueness 
hypothesis (ii) that the only common element of $K_{i}$ and $K_{j}$ is $P$. We wish to show that the $d$ grand cliques $K_{1}, K_{2}, \cdots, K_{d}$ are the only grand cliques containing $P$.

We begin by recalling that $P, S$ is a claw of order $d$ and that (in the notation of Lemma 4.3) the set

$$
H=H_{1} \cup H_{2} \cup \cdots \cup H_{d}
$$

consists of $S$ and of all vertices in $T$ which are joined to exactly one of the vertices $A_{1}, \cdots, A_{d}$ of $S$ and are also joined to $P$. That is (when we take $s=d$ in Lemma 4.3),

$$
|H|=f(1)+d \text {. }
$$

Moreover, since $G$ has no cliques of order $d+1, f(0)=0$. Thus (4.11), (4.12), with $s=d$, can be rewritten as

$$
\begin{aligned}
& |H|+\sum_{2}^{d} f(x)=d(n-1), \\
& \sum_{2}^{d}(x-1) f(x)=d(d-1)(d-2) .
\end{aligned}
$$

If $d=1$, the summation disappears in (4.25) and the inequalities

$$
d[n-1-(d-1)(d-2)] \leqq|H| \leqq d[n-1-(d-2)]
$$

hold trivially. If $d>1$, (4.26) yields

$$
\sum_{2}^{d} f(x) \leqq d(d-1)(d-2) \leqq(d-1) \sum_{2}^{d} f(x),
$$

whence

$$
-d(d-1)(d-2) \leqq-\sum_{2}^{d} f(x) \leqq-d(d-2) .
$$

The latter inequalities, combined with (4.25), yield (4.27).

Now let us suppose that $P$ is contained in at least one grand clique $K$ distinct from $K_{1}, K_{2}, \cdots K_{d}$. Then each of the $d(n-1)$. vertices (distinct from $P$ ) which are joined to $P$, is contained in at most one of the $d+1$ grand cliques. Moreover, $K_{1}, \cdots, K_{d}$ together contain at least $|H|$ of these vertices, and $K$, being a grand clique, contains at least

$$
n-1-(d-1)^{2}(d-2)
$$

more. Therefore, by (4.27),

$$
d[n-1-(d-1)(d-2)]+n-1-(d-1)^{2}(d-2) \leqq d(n-1)
$$


and hence (see (4.24))

$$
n-1 \leqq(d-1)^{2}(d-2)+d(d-1)(d-2)=q(d-1)-1 .
$$

This yields $n \leqq q(d-1)$, in contradiction to our hypothesis.

At this stage we have proved that each vertex $P$ lies in exactly $d$ distinct grand cliques $K_{1}, \cdots, K_{d}$. If $Q$ were a vertex joined to $P$ but in none of $K_{1}, \cdots, K_{d}$, then, by Lemma 4.6 , there would be a grand clique $K$, distinct from $K_{1}, \cdots, K_{d}$, containing $P$ and $Q$. Hence each of the $d(n-1)$ vertices joined to $P$ lies in one (and only one, by uniqueness) of $K_{1}, \cdots, K_{d}$. By Lemma 4.1, no maximal clique can have more than $n$ elements. Consequently, each of $K_{1}, \cdots, K_{d}$ must contain exactly $n$ vertices. That is, each $K_{i}$ is a line of $G$.

If $K$ is any grand clique of $G$, we fix attention on a vertex $P$ contained in $K$ and use the fact, just proved, that every grand clique containing $P$ is a line. Hence $K$ is a line of $G$. This completes the proof of Lemma 4.7.

We did not need the upper bound in (4.27) for the proof of Lemma 4.7. This upper bound shows, however, that, for $d>2$, the major cliques $\{P\} \cup H_{i}$ constructed in the proof are not all lines-else the upper bound would have to be at least $d(n-1)$.

Now we shall state and prove three theorems-three, because the varying hypotheses apply to different classes of graphs. We also state (4.2), which we have tacitly assumed up until this point.

THEOREM 4.1. Let $G$ be a pseudo net-graph of order $n$, degree $d$, with $n \geqq d \geqq 1$, which is subject to the following conditions: (i) $G$ has no claws of order $d+1$; (ii) two distinct grand cliques of $G$ have at most one common point; (iii) $n>q(d-1)$, where the polynomial $q$ is given by (4.21). Then $G$ is the graph of one and only one net of order $n$, degree $d$.

CoRollary. Assume, in addition to the hypotheses of Theorem 4.1, that $G$ is the complementary graph of a nontrivial net $N$ of order $n$, deficiency $d$. Then $N$ can be imbedded uniquely in an affine plane $\pi$ of order $n$, and $G$ is graph of the net complementary to $N$ in $\pi$.

Proof. We may apply Lemma 4.7. Since every grand clique of $G$ is a line, we see that each vertex of $G$ lies on exactly $d$ distinct lines of $G$. Let $P$ be a vertex of $G$ and let $L$ be a line of $G$ not containing $P$. By Lemma 4.1 (i), $P$ is joined to exactly $d-1$ distinct vertices in $L$. By Lemma 4.6 (which we may apply in view of (4.24)) these $d-1$ vertices lie one each on $d-1$ lines through $P$. Thus there is one and only one line, $L^{\prime}$, through $P$ which is parallel to $L$ 
(has no vertex in common with $L$.) If we choose any line $M$ which meets $L$, we see that through each vertex in $M$ but not $L$ there passes a unique line parallel to $L$. By uniqueness, no two such parallels can intersect. Hence $L$ determines a parallel class $L$ consisting of $n$ lines, including $L$ itself, each two of which are parallel. It is now clear that the vertices of $G$ (considered as points) and the lines of $G$ constitute a net of order $n$, degree $d$. Since two distinct. vertices of $G$ are joined in $G$ if and only if they lie on a common line of $G$, we see that $G$ is the graph of the net. The Corollary is immediate, in view of the discussion in $\S 3$. This completes the proof of Theorem 4.1 and Corollary.

THEOREM 4.2. Let $G$ be a pseudo net-graph of order $n$, degree d. such that (i) $G$ has no claws of order $d+1$ and (ii) $n>2(d-1)^{3} \geqq 0$ (and, in case $d=1$, also $n>1$.) Then $G$ is the graph of one and only one net of order $n$, degree $d$.

Corollary. Assume, in addition to the hypotheses of Theorem 4.2, that $G$ is the complementary graph of a net $N$ of order $n$, deficiency $d$. Then $N$ can be imbedded uniquely in an affine plane. $\pi$ of order $n$, and $G$ is the graph of the net complementary to $N$ in $\pi$.

Proof. We need merely show that the hypotheses of Theorem 4.1 are verified. Hypothesis (i) of Theorem 4.2 is identical with hypothesis (i) of Theorem 4.1. In view of the Corollary of Lemma 4.2, hypothesis (ii) of Theorem 4.2 implies hypothesis (ii) of Theorem 4.1. Since

$$
2(d-1)^{3}-q(d-1)=(d-1)^{2}+(d-1)-1>0 \quad \text { for } d>1
$$

and

$$
q(0)=1 \text {, }
$$

hypothesis (ii) of Theorem 4.2 also implies hypothesis (iii) of Theorem: 4.1. This completes the proof.

THEOREM 4.3. Let $G$ be a pseudo net-graph of order $n$, degree $d$ such that $n>p(d-1)$, where $p$ is the polynomial given by (4.17), and either $d=1, n>1$ or $d>1$. Then $G$ is the graph of one and. only one net of order $n$, degree $d$.

Corollary. Assume, in addition to the hypotheses of Theorem. 4.3, that $G$ is the complementary graph of a net $N$ of order $n$, deficiency $d$. Then $N$ can be imbedded uniquely in an affine plane 
$\pi$ of order $n$, and $G$ is the graph of the net complementary to $N$ in $\pi$.

Proof. $p(0)=q(0)=1$ and

$$
p(d-1)-2(d-1)^{3}=\frac{1}{2}\left\{(d-1)^{3}(d-3)+2(d-1)^{2}+3(d-1)\right\}>0
$$

if $d>1$. Thus we may apply Lemma 4.4 to get the hypotheses of Theorem 4.2.

5. Incidence matrices. We are going to show that certain sets of $k$ mutually orthogonal symmetric matrices of order $n^{2}$ are closely akin to nets of order $n$, degree $k$-and thus to sets of $k-2$ mutually orthogonal latin squares of side $n$. Surprising as it may seem, in view of the coincidence of the adjective "orthogonal" in "orthogonal matrices" and "orthogonal latin squares," we have it on the authority of R. C. Bose that, when orthogonal latin squares are used in the analysis of statistics, no orthogonal matrices arise such as the ones here defined. Thus the correspondence seems to be new.

It will be convenient to have a name for the matrices we study, and we adopt the adjective "germaine" as a pseudonym for "akin." By a germaine matrix, $F$, of order $n^{2}$ we mean a matrix $F$ of $n^{2}$ rows and columns such that (i) $F$ is symmetric; (ii) every entry on the main diagonal of $F$ is $n-1$; (iii) every other entry of $F$ is either $n-1$ or -1 ; (iv) $F^{2}=n^{2} F$.

If $F$ is a germaine matrix of order $n^{2}$, then, by (iv), the matrix $E=n^{-2} F$ is idempotent and, by (ii), $E$ has trace $n-1$. Since (over a field of characteristic zero) the trace of an idempotent matrix is equal to its rank, we see that $E$ and $F$ have rank $n-1$. When $n=2$, there are germaine matrices of order 4 which we want to avoid, e.g., the matrix with every entry equal to 1 . This is an exception to the general rule (which will be clear in a moment) that germaine matrices have zero row-sums.

In order to avoid complications of notation, we begin with two lemmas concerning one and two germaine matrices respectively.

LEMMA 5.1. Let $n \geqq 2$ be an integer. To each enumeration $1,2, \cdots, n^{2}$ of the $n^{2}$ points of a net $N$ of order $n$, degree 1 there corresponds a germaine matrix $F$ of order $n^{2}$ defined as follows: $F$ has $n-1$ down the main diagonal; for $i \neq j, F$ has $n-1$ or -1 in position $(i, j)$ according as the points $i, j$ of $N$ lie or do not lie on a line of $N$. Moreover, $F$ has zero row sums. Conversely, if $F$ is a germaine matrix of order $n^{2}$ (and if, in case $n=2, F$ has zero row sums) then $F$ arises from a net $N$ of order $n$, degree 1 in the manner indicated. 
Proof. If $N$ is given, and if $F$ is defined from $N$ as described, it is a straightforward matter to verify that $F$ is germaine and has zero row-sums. Conversely, let

$$
F=\left(f_{i j}\right)
$$

be a given germaine matrix of order $n^{2}$ (with zero row-sums in case $n=2$ ). Since $F$ is symmetric, condition (iv) for a germaine matrix may be written as

$$
n^{2} f_{i j}=\sum_{k} f_{i k} f_{j k} \quad\left(i, j=1,2, \cdots, n^{2}\right)
$$

where $k$ ranges from 1 to $n^{2}$. Let $G$ be a graph whose $n^{2}$ vertices are the integers $1,2, \cdots, n^{2}$. For $i \neq j$, let vertices $i, j$ form an edge if and only if $f_{i j}=n-1$. Since $n-1 \neq-1$, the edges of $F$ are well-defined, by (ii), (iii). Moreover, by (i), $G$ is symmetric.

Our first task is to show that $G$ is a pseudo net-graph of order $n$, degree $d=1$. Thus, in the sense of $\S 4$, we must show that

$$
n_{1}=n-1, \quad p_{11}^{1}=n-2, \quad p_{11}^{2}=0 .
$$

Consider some fixed vertex $i$ of $G$ and suppose that $i$ is joined to $x$ and not joined to $n^{2}-1-x$ of the remaining $n^{2}-1$ vertices. Taking $j=i$ in (5.1), and using the properties of $F$, we get

$$
n^{2}(n-1)=(n-1)^{2}(1+x)+1 \cdot\left(n^{2}-1-x\right),
$$

whence

$$
n^{2}(n-2)=n(n-2)(1+x) .
$$

If $n>2$, we get $1+x=n, x=n-1$. In any case, the sum of the $i$ th row of $F$ is

$$
(n-1)(1+x)-\left(n^{2}-1-x\right)=n(x-n+1),
$$

and this is zero precisely when $x=n-1$. Therefore we have $n_{1}=$ $x=n-1$, whence

$$
n_{1}=n-1, \quad n_{2}=n^{2}-n,
$$

where, of the $n^{2}-1$ vertices distinct from $i, n_{1}$ are joined and $n_{2}$ are not joined to $i$. Next consider two distinct vertices $i, j$, joined in $G$. Of the $n^{2}-2$ vertices distinct from $i, j$ let $p_{11}^{1}=y$ be joined to both $i$ and $j$. Then (since $i, j$ are joined) $p_{12}^{1}=n_{1}-1-y=n-$ $2-y$ are joined to $i$ but not $j$, and $p_{21}^{1}=n-2-y$ are joined to $j$ but not $i$, and $p_{22}^{1}=n_{2}-p_{21}^{1}=n^{2}-2 n+2+y$ are joined to neither $i$ nor $j$. Using (5.1) for the given $i, j$, we get 


$$
\begin{aligned}
n^{2}(n-1) & =2(n-1)^{2}+(n-1)^{2} p_{11}^{1}-(n-1)\left(p_{12}^{1}+p_{21}^{1}\right)+p_{22}^{1} \\
& =n^{2}+n^{2} y
\end{aligned}
$$

whence $y=n-2$. Thus

$$
p_{11}^{1}=n-2, \quad p_{12}^{1}=p_{21}^{1}=0, \quad p_{22}^{1}=n(n-1) .
$$

Finally, consider two distinct vertices $i, j$ not joined in $G$. This time we may set

$$
\begin{aligned}
& p_{11}^{2}=z, \quad p_{12}^{2}=p_{12}^{2}=n_{1}-z=n-1-z, \\
& p_{22}^{2}=n_{2}-1-p_{21}^{2}=n^{2}-2 n+z .
\end{aligned}
$$

From (5.1) we get

$$
\begin{aligned}
n^{2}(-1) & =2(n-1)(-1)+(n-1)^{2} p_{11}^{2}-(n-1)\left(p_{12}^{2}+p_{21}^{2}\right)+p_{22}^{2} \\
& =-n^{2}+n^{2} z .
\end{aligned}
$$

Therefore $z=0$ and

$$
p_{11}^{2}=0, \quad p_{12}^{2}=p_{21}^{2}=n-1, \quad p_{22}^{2}=n-2+(n-1)(n-2) .
$$

This completes the proof that $G$ is a pseudo net-graph of order $n$, degree $d=1$, deficiency $k=n$.

Since $p(0)=0$ and since $d=1, n>1$, we may conclude from Theorem 4.3 that $G$ is the graph of a net $N$ of order $n$, degree $d=1$. This completes the proof of Lemma 5.1.

We recall that two matrices $A, B$ are orthogonal provided $A B=$ $B A=0=$ the zero matrix.

Lemma 5.2. Let $n \geqq 2$ be an integer. To each enumeration $1,2, \cdots, n^{2}$ of the $n^{2}$ points of a net $N$ of order $n$, degree 2 , and to each enumeration 1, 2 of the two line-classes of $N$, there corresponds an ordered pair $F_{1}, F_{2}$ of orthogonal germaine matrices of order $n^{2}$, such that, for $\alpha=1,2, F_{a}$ corresponds in the sense of Lemma 5.1 to the net $N_{a}$ of order $n$, degree 1 with the same points as $N$ and with the lines of class $\alpha$ as its lines. Conversely, if $F_{1}, F_{2}$ is an ordered pair of orthogonal germaine matrices, (with zero row sums, in case $n=2)$ then $F_{1}, F_{2}$ arises from a net $N$ of order $n$, degree 2 in the manner indicated.

Proof. If $N$ is given, and if $F_{1}, F_{2}$ are defined from $N$ as described, then $F_{1}, F_{2}$ are germaine by Lemma 5.1, and, by a straightforward computation, $F_{1}, F_{2}$ are orthogonal. Now we assume, conversely, that $F_{1}, F_{2}$ are orthogonal and germaine. By Lemma 5.1, for $\alpha=1,2, F_{\alpha}$ defines a net $N_{\alpha}$ of order $n$, degree 1 on the points $1,2, \cdots, n^{2}$. We let $N$ be the system with the same points as $N_{1}$ and 
$N_{2}$ and with the lines of $N_{1}$ as its first parallel class and the lines of $N_{2}$ as its second parallel class. Then each line of $N$ contains exactly $n$ distinct points, and each point of $N$ lies on exactly one line of each class. To prove that $N$ is a net of order $n$, degree 2 , we need only show that two lines of distinct classes have one and only one common point.

If we write $F_{1}=\left(a_{i j}\right), F_{2}=\left(b_{i j}\right)$, then, since $F_{1}, F_{2}$ are symmetric, orthogonality is expressed by the condition

$$
\sum_{k} a_{i k} b_{j k}=0 \quad\left(i, j=1,2, \cdots, n^{2}\right) .
$$

First we consider a point $i$ and suppose that, of the $n^{2}-1$ other points, $x_{11}$ are joined to $i$ both by a line of class 1 and a line of class. $2, x_{10}$ are joined to $i$ by a line of class 1 but not by a line of class. 2 , and similarly for $x_{01}, x_{00}$. Setting $x_{11}=x$, we see that

$$
x_{10}=x_{01}=n-1-x, \quad x_{00}=n^{2}-2 n+1+x .
$$

From (5.6) with $i=j$, we get (since $F_{1}, F_{2}$ are germaine)

$$
\begin{aligned}
0 & =(n-1)^{2}\left(1+x_{11}\right)-(n-1)\left(x_{10}+x_{01}\right)+x_{00} \\
& =n^{2} x .
\end{aligned}
$$

Hence $x=0$. That is, if two lines of distinct classes have a common point $i$, then they have no other common point.

There remains the possibility that there are two lines of different. classes with no common point. Suppose that, for some $i \neq j$, the line of class 1 through $i$ and the line of class 2 through $j$ have no common point. Then, from (5.6), for the given $i, j$, we get

$$
\begin{aligned}
0 & =n(n-1)(-1)+n(n-1)(-1)+\left[\left(n^{2}-2 n\right)(-1)^{2}\right] \\
& =-n^{2},
\end{aligned}
$$

a contradiction. This completes the proof of Lemma 5.2.

Now we are ready for the main theorem.

THeonem 5.1. Let $n, k$ be integers, with $n \geqq 2,1 \leqq k \leqq n+1$. To each enumeration $1,2, \cdots, n^{2}$ of the $n^{2}$ points of a net $N$ of order $n$, degree $k$, and to each enumeration $1,2, \cdots, k$ of the $k$ line-classes of $N$, there corresponds an ordered set

$$
F_{1}, F_{2}, \cdots, F_{k}
$$

of mutually orthogonal germaine matrices of order $n^{2}$ such that, for $\alpha=1,2, \cdots, k, F_{a}$ corresponds in the sense of Lemma 5.1 to the net $N_{\alpha}$ of order $n$, degree 1 with the same points as $N$ and with the lines of class $\alpha$ as its lines. Conversely, if (5.7) is an ordered set 
of $k$ mutually orthogonal germaine matrices of order $n^{2}$ (each with zero row-sums, in case $n=2$ ) then (5.7) arises from a net $N$ of order $n$, degree $k$ in the manner indicated.

Proof. In view of Lemmas 5.1, 5.2, we need only treat the case $k \geqq 3$. In this case, for $\alpha<\beta$, if $N$ is given, let $N_{\alpha \beta}$ be the net of order $n$, degree 2 with the same points as $N$ and with the line-classes $\alpha, \beta$ as its two line-classes. By Lemma 5.2, $N_{\alpha \beta}$ determines the ordered pair $F_{\alpha}, F_{\beta}$ of orthogonal germaine matrices of order $n^{2}$. Conversely, the pair determines $N_{\alpha \beta}$. It should now be clear that the set (5.7) determines a net $N$ of order $n$, degree $k$. Indeed, the only point which could be at issue is whether two lines of distinct classes in $N$ have a unique common point, and this follows from the fact that each $N_{\alpha \beta}$ is a net of degree 2. The proof of Theorem 5.1 is now complete.

For a (non-trivial) net $N$ of order $n$, degree $k$, deficiency $d$ we also define matrices $F_{0}, F^{*}, F_{\infty}$ in addition to (5.7). First we define

$$
F^{*}=\sum_{i=1}^{k} F_{i}
$$

In addition, $F_{0}$ (usually called $J$ or $S$ ) is the matrix of order $n^{2}$ with every entry equal to 1 , and, finally, $F_{\infty}$ is defined by the equation

$$
n^{2} I=F_{0}+F^{*}+F_{\infty},
$$

where $I$ is the identity matrix of order $n^{2}$. We shall give a direct description of $F^{*}$ and $F_{\infty}$ : The matrix $F^{*}$ has $k(n-1)$ down the main diagonal and, for $i \neq j$, has $d-1$ or $-k$ in place $(i, j)$ according as the points $i, j$ of $N$ are joined in $N$ (by a line of any class) or not joined in $N$. By contrast, the matrix $F_{\infty}$ has $d(n-1)$ down the main diagonal and, for $i \neq j$, has $-d$ or $k-1$ in place $(i, j)$ according as the points $i, j$ are joined or not joined in $N$. Clearly it is reasonable to associate $F^{*}$ with the graph $G_{1}$ of $N$ and $F_{\infty}$ with the complementary graph $G_{2}$. We note that if $N$ is an affine plane, so that $d=0$ and every two points of $N$ are joined, then $F_{\infty}$ is the zero matrix, and $F^{*}$ has $n^{2}-1$ down the main diagonal, -1 off the main diagonal.

If the graph $G$ of the theorem which follows is the complementary graph of the above net $N$, then the matrix $F$ of the theorem is $F_{\infty}$ (except that the words "joined" and "not joined" have been interchanged):

TheOREM 5.2. Let $n, d, k$ be positive integers with

$$
d+k=n+1 .
$$


To each ordering $1,2, \cdots, n^{2}$ of the $n^{2}$ vertices of a pseudo net-graph $G$ of order $n$, degree $d$, deficiency $k$ there corresponds a matrix $F$ of order $n^{2}$ with the following properties: (i) $F$ is symmetric; every entry in the main diagonal of $F$ is $d(n-1)$; (iii) every other entry of $F$ is either $k-1$ or $-d$. (iv) $F^{2}=n^{2} F$. Specifically, we define $F$ by insisting on (ii) and, for distinct vertices $i, j$, by putting $k-1$ or $-d$ in place $(i, j)$ of $F$ according as $i, j$ are joined or not joined in $G$. The matrix $F$, so defined, has zero row sums. Conversely, if $F$ is a matrix of order $n^{2}$ with properties (i)-(iv) (and if $F$ has zero row-sums in case $n=2 d$ ), then $F$ arises from a pseudo net-graph $G$ of order $n$, degree $d$, deficiency $k$ in the manner indicated.

Sketch of proof. We note that, when $d=1$, Theorem 5.2 is essentially Lemma 5.1 (stated for a graph instead of a net). The direct part of the proof is straightforward and the converse part can be stated so as to reduce to the main part of the proof of Lemma 5.1 when $d=1$. The only difference is that we do not claim-and, for $n$ small compared with $d$, we cannot claim - that $F$ determines a net. This should suffice for the proof of Theorem 5.2.

It should be observed that if the edges of the graph $G$ of Theorem 5.2 can be partitioned into two sets so that $G$ can be regarded as made up of two graphs $G_{1}, G_{2}$ on the same vertices, where $G_{\alpha}$ is a pseudo net-graph of order $n$, degree $d_{\alpha}$ (and $d=d_{1}+d_{2}$ ) then the matrix $F$ of Theorem 5.2 can be decomposed $\left(F=F_{1}+F_{2}\right)$ into the sum of a pair of orthogonal matrices $F_{1}, F_{2}$, where $F_{\alpha}$ is defined for $G_{a}$ in the manner of Theorem 5.2. Precisely when $G$ is a net-graph, $F$ can be decomposed into a sum of $d$ mutually orthogonal germaine matrices.

Returning again to a net $N$ of order $n$, degree $k$, deficiency $d$, and to the matrices exhibited in (5.8), (5.9), we wish to discuss briefly point-point incidence matrices for $N$. First we define

$$
E_{i}=n^{-2} F_{i}(0 \leqq i \leqq k), \quad E^{*}=n^{-2} F^{*}, \quad E_{\infty}=n^{-2} F_{\infty}
$$

and observe that these $E$ 's are mutually orthogonal idempotent matrices. Moreover

$$
\begin{gathered}
E^{*}=\sum_{i=1}^{k} E_{i}, \\
I=E_{0}+E^{*}+E_{\infty}, \\
\operatorname{rank} E_{0}=1, \quad \operatorname{rank} E_{i}=n-1(1 \leqq i \leqq k), \\
\operatorname{rank} E^{*}=k(n-1), \quad \operatorname{rank} E_{\infty}=d(n-1) .
\end{gathered}
$$

Next let 


$$
x, y_{1}, \cdots, y_{k}, z
$$

be $k+2$ rational numbers and define the point-point incidence matrix

$$
A\left(x, y_{1}, \cdots, y_{k}, z\right)=(a(i, j))
$$

of order $n^{2}$ as follows: $a(i, i)=x$ for all $i$; if $i \neq j$, and if the points $i, j$ of $N$ lie on a line of class $\alpha, a(i, j)=y_{\alpha}$; if $i \neq j$, and if the points $i, j$ are not joined in $N, a(i, j)=z$. We may express the $F$ 's and $E$ 's in terms of $A\left(x, y_{1}, \cdots, y_{k}, z\right)$ for suitable choices of $x$, the $y$ 's and $z$. Specifically, we get $F_{0}$ by taking $x$, the $y$ 's and $z$ all equal to 1 . We get $F_{\alpha}(1 \leqq \alpha \leqq k)$ by taking $x=y_{\alpha}=n-1, z=-1$ and $y_{\beta}=-1$ for $\beta \neq \alpha$. And we get $F_{\infty}$ by taking $x=d(n-1), y_{\alpha}=$ $-d(1 \leqq \alpha \leqq k)$ and $z=k-1$. Conversely, we may easily verify that

$$
A\left(x, y_{1}, \cdots, y_{k}, z\right)=X E_{0}+\sum_{i=1}^{k} Y_{i} E_{i}+Z E_{\infty}
$$

where

$$
\begin{aligned}
X & =x+(n-1) y^{*}+d(n-1) y, \\
Y_{i} & =x+n y_{i}-y^{*}-d z, \\
Z & =x-y^{*}+(k-1) z,
\end{aligned}
$$$$
(i \leqq i \leqq k)
$$

Since the $E$ 's are mutually orthogonal idempotents of known ranks, we see at once that the characteristic roots of $A\left(x_{1}, y_{1}, \cdots, y_{k}, z\right)$ are: $X$ of multiplicity 1; (for $1 \leqq i \leqq k) \quad Y_{i}$ of multiplicity $n-1$; and $Z$ of multiplicity $d(n-1)$. - For certain choices of $x$, the $y$ 's and $z$, some of these roots coincide; then their multiplicities must be added.

The results of the preceding paragraph may be used to show that a conjecture originally advanced by Harary and later disproved by Bose (along the present lines), is quite impossible to repair. We recall that, for any finite symmetric graph $G$ with $s$ vertices $1,2, \cdots, s$, the adjacency matrix of $G$ is a matrix of order $s$ with 0 down the main diagonal, and with 1 or 0 in non-diagonal position $(i, j)$ according as the vertices $i, j$ are joined or not joined in $G$. Harary's conjecture was that (to within an isomorphism) a finite symmetric graph was determined by the characteristic roots of its adjacency matrix, taken with their multiplicities. However, if $G_{1}, G_{2}$ are the graph and the complementary graph of the net $N$ of the preceding paragraph, the adjacency matrix of $G_{1}$ comes from (5.16) by taking $x=z=0$ and all $y$ 's equal to 1 , and the adjacency matrix of $G_{2}$ comes by taking $x=0, z=1$ and all $y$ 's equal to 0 . In either case, the characteristic roots and their multiplicities depend only upon $n, k$ and $d$. Let us 
concentrate on $G_{1}$ and note, from Theorem 4.3, that, if $n>p(k-1)$, $G_{1}$ uniquely determines $N$. It follows that, for $n>p(k-1)$, there are precisely as many graphs $G_{1}$ as there are nets $N$. However, even in the special case $k=3$, corresponding to a single latin square, the number of nets of order $n$, degree $k$ increases astronomically with $n$. (Cf. Hall [7].)

Finally, we wish to mention line-point incidence matrices, again for the same net $N$. With each line $L$ of the net $N$ we associate a row-vector of $n^{2}$ columns, having 1 or 0 in column $i$ according as point $i$ lies or does not lie on $L$. With the $\alpha$ th line class of $N$ we associate a matrix $M_{\alpha}$ of $n$ rows, $n^{2}$ columns, the rows of $M_{\alpha}$ being those for the lines of class $\alpha$, in any order. Finally, we define $M$ to be the matrix of $k n$ rows, $n^{2}$ columns, given by

$$
M=\left(\begin{array}{c}
M_{1} \\
M_{2} \\
\vdots \\
M_{k}
\end{array}\right)
$$

Then $M$ is the line-point incidence matrix of $N$. We merely wish to note that, where $A^{T}$ denotes the transpose of matrix $A$,

$$
\begin{aligned}
M_{\alpha}^{T} M_{\alpha} & =n E_{0}+n E_{\alpha}, & 1 \leqq \alpha \leqq k, \\
M^{r} M & =k n E_{0}+n E^{*} . &
\end{aligned}
$$

As a consequence, the germaine matrices of Theorem 5.1 bear a simple relationship to the matrices $M_{\alpha}^{T} M_{\alpha}$.

6. Remarks and examples. In [4] we assigned to each non-trivial non-degenerate net $N$ of order $n$, degree $k$ (and deficiency $d$ ) a numerical invariant $\phi(N)$ with properties like Euler's totient. In particular, a necessary condition for the existence for a transversal to $N$ is that $\phi(N)=1$. (Consequently, by Theorem $4.3, \phi(N)=1$ if $n>p(d-1)$.)

It was remarked in [4] that the necessary condition $\phi(N)=1$ was not sufficient for the existence of a transversal, but this statement seems to have been missed. Accordingly, we remark here that if $N$ is the net of the latin square

$\begin{array}{llllll}1 & 2 & 3 & 4 & 5 & 6 \\ 2 & 3 & 4 & 5 & 6 & 1 \\ 3 & 5 & 1 & 6 & 2 & 4 \\ 4 & 6 & 2 & 1 & 3 & 5 \\ 5 & 4 & 6 & 2 & 1 & 3 \\ 6 & 1 & 5 & 3 & 4 & 2\end{array}$


then $\phi(N)=1$ but $N$ has no transversals. - This example recently disproved a conjecture and stopped a computer program.

If $G$ is the complementary graph of the net of (6.1), then $G$ has order $n=6$, degree $d=4$, deficiency $k=3$, and $G$ has no lines whatever. For other examples of this type, we need the MacNeish number, $M(n)$, of the positive integer $n$ : If $n$ is a prime-power, $M(n)=n$. If $n$ is a product of prime-powers involving distinct primes, then $M(n)$ is the least of these prime powers. MacNeish showed that, for every positive integer $n \geqq 2$, there is at least one set of $M(n)-1$ mutually orthogonal latin of side $n$ (thus, a net of order $n$, degree $M(n)+1$ ) and he conjectured (incorrectly) that there could be no more. In [4], using a direct product construction essentially due to MacNeish, we showed rather more: For each positive integer $n$ there exists at least one net $N$ of order $n$, degree $k=M(n)+1$, deficiency $d=n-M(n)$, for which $\phi(N)=M(n)>1$. Such a net $N$, of course, has no transversals. Thus, if $G$ is the complementary graph of $N, G$ is a pseudo net-graph of order $n$, degree $D(n)=n-M(n)$ with no lines whatever. If $n$ is a prime-power, $D(n)=0$ and the result has no interest. If $n=P Q$ where $P$ is a prime-power, $Q$ is prime to $P$, and $P$ is the least prime-power dividing $n$, then $M(n)=P$ and $n / D(n)=Q /(Q-1)$. Hence, for $Q$ large, $D(n)$ is close to $n$. For example, $D(20)=16$. Thus these examples are of little help with the theorems of $\S \S 3,4$, though they do show that some conditions are necessary.

We call attention to other examples briefly discussed in $\S 3$.

The results of the present paper, especially Theorems 3.1 and Theorems 4.1, 4.2, 4.3, suggest that a further study of pseudo netgraphs of order $n$, degree $d$ subject to

$$
d>1, n>d+1,(d-1)^{2}<n \leqq p(d-1)
$$

would be rewarding. We offer the following:

Conjecture. Every pseudo net-graph of order $n$, degree $d$, deficiency $k$, subject to (6.2), is either the graph of a net of order $n$, degree $d$ or the complementary graph of a net of order $n$, degree ke or both.

When $d=2$, (6.2) yields $n=4$, whence $k=3$. As Shrikhande shows, there are just two pseudo net-graphs of order 4, degree 2, deficiency 3. One comes from the plane of order 4 and is thus both a net-graph and a complementary net-graph. The other is not a netgraph but is the complementary graph of the net of order 4, degree 3 defined by the cyclic group of order 4 . The situation for $d>2$ is completely unknown to the author, except for $n \leqq 7$. 
One of the difficulties in dealing with pseudo net-graphs is the lack of a method of forming a "direct product" of two of them in such a way as to end up with a pseudo net-graph. The direct product of two nets (and hence of two net-graphs) of the same degree is easily defined (cf. [4]) but uses the existence of line-classes. This construction is unavailable for pseudo net-graphs. The direct-product construction for nets of the same degree allows a direct-product construction for complementary net-graphs of the same deficiency-but here we require too much knowledge of the nets to permit a generalization.

AdDendum. Dale Mesner, in his unpublished $\mathrm{Ph}$. D. thesis ("An investigation of certain combinatorial properties of partially balanced incomplete block experimental designs and association schemes, with a detailed study of designs of Latin squares and related topics," Michigan State University, 1956) has results allied to Theorem 4.3. Essentially, he proves Theorem 4.3 with the hypothesis $n>p(d-1)$ replaced by a stronger hypothesis $n>d_{0}$. Here we may define $d_{0}$ to be the greatest integer in the largest of the real roots obtained from the quadratic equations

$$
\begin{aligned}
& 4 x^{2}-(d-1)\left(9 d^{2}-9 d+7\right) x+(d-1)^{2}\left(9 d^{2}-9 d+7\right)=0, \\
& 2 d x^{2}-\left(d^{5}-2 d^{4}+3 d^{3}-d^{2}-2 d+1\right) x \\
& \quad-\left(d^{6}-3 d^{5}+3 d^{4}+2 d^{3}-3 d^{2}+d+1\right)=0 .
\end{aligned}
$$

With a little labor we may verify the inequalities

$$
\frac{1}{2} d^{4}-d^{3}<p(d-1)<d_{0}<\frac{1}{2} d^{4}, \quad d \geqq 2,
$$

which show that Mesner's result is close to Theorem 4.3 but not as sharp.

\section{BIBLIOGRAPHY}

1. R. C. Bose, S. S. Shrikhande and E. T. Parker, Further results on the construction of mutually orthogonal latin squares and the falsity of Euler's conjecture Canad. J. Math., 12 (1960), 189-203.

2. R. C. Bose and T. Shimamoto, Classification and analysis of partially balanced incomplete block designs with two asseciate classes, J. Amer. Stat. Assn., 47 (1952), 151-184.

3. R. H. Bruck and H. J. Ryser, The non-existence of certain finite projective planes, Canad. J. Math., 1 (1949), 88-93.

4. R. H. Bruck, Finite Nets, I. Numerical Invariants, Canad. J. Math., 3 (1951) 94107.

5. S. Chowla, P. Erdos and E. G. Straus, On the maximal number of pairwise orthogonal latin squares of a given order, Canad. J. Math., 12 (1960), 204-208.

6. R. A. Fisher and F. Yates, Statistical Tables for Agricaltural, Biological and Medical Research, (Edinburgh, 1943).

7. Marshall Hall, Jr. Distinct representatives of subsets, Bull. Amer. Math. Soc., 54 (1948), 922-926. 
8. H. W. Norton, The $7 \times 7$ squares, Ann. Eugen. 9 (1939), 269-307.

9. S. S. Shrikhande, $A$ note on mutually orthogonal latin squares, Sankya (Indian Journal), 23 (1961), 115-116.

10. - The uniqueness of the $L_{2}$ association scheme, Annals of Math. Stat., 30 (1959), 781-798.

UNIVERSITY OF WISCONSIN

MADISON 6, WISCONSIN 



\title{
PACIFIC JOURNAL OF MATHEMATICS
}

\author{
EDITORS
}

\author{
Ralph S. Phillips \\ Stanford University \\ Stanford, California \\ M. G. Arsove \\ University of Washington \\ Seattle 5 , Washington
}

\author{
J. DugunduI \\ University of Southern California \\ Los Angeles 7, California
}

Lowell J. Paige

University of California

Los Angeles 24, California

\section{ASSOCIATE EDITORS}

E. F. BECKENBACH

T. M. CHERRY
D. DERRY
M. OHTSUKA

H. L. ROYDEN

E. SPANIER
E. G. STRAUS

F. WOLF

\section{SUPPORTING INSTITUTIONS}

UNIVERSITY OF BRITISH COLUMBIA CALIFORNIA INSTITUTE OF TECHNOLOGY UNIVERSITY OF CALIFORNIA MONTANA STATE UNIVERSITY UNIVERSITY OF NEVADA NEW MEXICO STATE UNIVERSITY OREGON STATE UNIVERSITY UNIVERSITY OF OREGON OSAKA UNIVERSITY UNIVERSITY OF SOUTHERN CALIFORNIA
STANFORD UNIVERSITY

UNIVERSITY OF TOKYO

UNIVERSITY OF UTAH

WASHINGTON STATE UNIVERSITY

UNIVERSITY OF WASHINGTON

AMERICAN MATHEMATICAL SOCIETY CALIFORNIA RESEARCH CORPORATION SPACE TECHNOLOGY LABORATORIES NAVAL ORDNANCE TEST STATION

Mathematical papers intended for publication in the Pacific Journal of Mathematics should be typewritten (double spaced), and the author should keep a complete copy. Manuscripts may be sent to any one of the four editors. All other communications to the editors should be addressed to the managing editor, L. J. Paige at the University of California, Los Angeles 24, California.

50 reprints per author of each article are furnished free of charge; additional copies may be obtained at cost in multiples of 50 .

The Pacific Journal of Mathematics is published quarterly, in March, June, September, and December. Effective with Volume 13 the price per volume (4 numbers) is $\$ 18.00$; single issues, $\$ 5.00$. Special price for current issues to individual faculty members of supporting institutions and to individual members of the American Mathematical Society: $\$ 8.00$ per volume; single issues \$2.50. Back numbers are available.

Subscriptions, orders for back numbers, and changes of address should be sent to Pacific Journal of Mathematics, 103 Highland Boulevard, Berkeley 8, California.

Printed at Kokusai Bunken Insatsusha (International Academic Printing Co., Ltd.), No. 6, 2-chome, Fujimi-cho, Chiyoda-ku, Tokyo, Japan.

PUBLISHED BY PACIFIC JOURNAL OF MATHEMATICS, A NON-PROFIT CORPORATION

The Supporting Institutions listed above contribute to the cost of publication of this Journal, but they are not owners or publishers and have no responsibility for its content or policies. 


\section{Pacific Journal of Mathematics}

\section{Vol. 13, No. 2 \\ April, 1963}

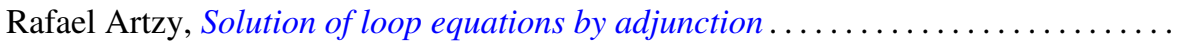

Earl Robert Berkson, A characterization of scalar type operators on reflexive

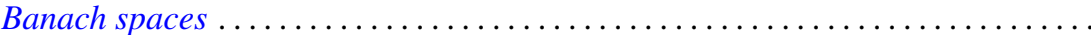

Mario Borelli, Divisorial varieties

365

Raj Chandra Bose, Strongly regular graphs, partial geometries and partially

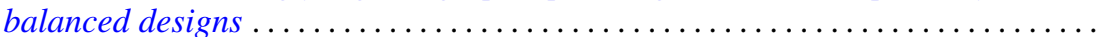

389

R. H. Bruck, Finite nets. II. Uniqueness and imbedding ............... 421

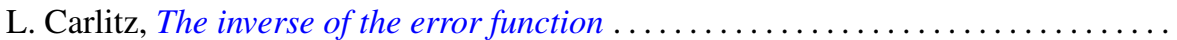

Robert Wayne Carroll, Some degenerate Cauchy problems with operator

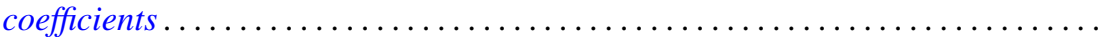

Michael P. Drazin and Emilie Virginia Haynsworth, A theorem on matrices of 0 's

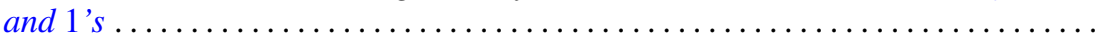

Lawrence Carl Eggan and Eugene A. Maier, On complex approximation .......... James Michael Gardner Fell, Weak containment and Kronecker products of group

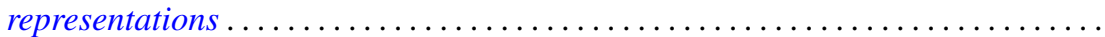

Paul Chase Fife, Schauder estimates under incomplete Hölder continuity

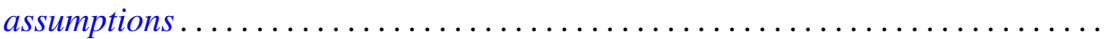

Shaul Foguel, Powers of a contraction in Hilbert space ...................

Neal Eugene Foland, The structure of the orbits and their limit sets in continuous

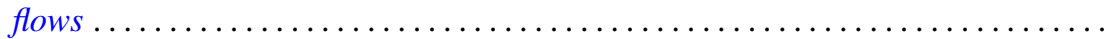

Frank John Forelli, Jr., Analytic measures . . . . . . . . . . . . . . . . . . . . . 563

Robert William Gilmer, Jr., On a classical theorem of Noether in ideal theory ....... P. R. Halmos and Jack E. McLaughlin, Partial isometries .

Albert Emerson Hurd, Maximum modulus algebras and local approximation in

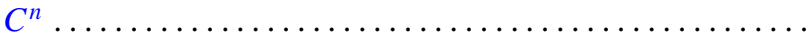

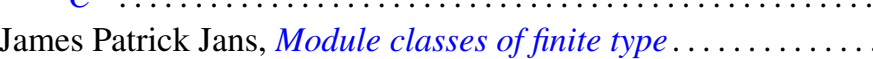

Betty Kvarda, On densities of sets of lattice points ...

H. Larcher, A geometric characterization for a class of discontinuous groups of linear fractional transformations .

John W. Moon and Leo Moser, Simple paths on polyhedra .

T. S. Motzkin and Ernst Gabor Straus, Representation of a point of a set as sum of

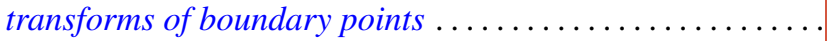

Rajakularaman Ponnuswami Pakshirajan, An analogue of Kolmogorov's three-series theorem for abstract random variables ...............

Robert Ralph Phelps, Čebyšev subspaces of finite codimension in $C(X)$...

James Dolan Reid, On subgroups of an Abelian group maximal disjoint from a given subgroup ...

William T. Reid, Riccati matrix differential equations and non-oscillation criteria for associated linear differential systems .................

Georg Johann Rieger, Some theorems on prime ideals in algebraic number fields ...

Gene Fuerst Rose and Joseph Silbert Ullian, Approximations of functions on the

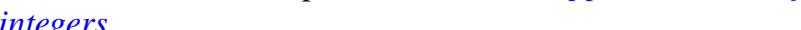

F. J. Sansone, Combinatorial functions and regressive isols . . . . . . . . . . 703

Leo Sario, On locally meromorphic functions with single-valued moduli . . . . . . . 709

Takayuki Tamura, Semigroups and their subsemigroup lattices.

Pui-kei Wong, Existence and asymptotic behavior of proper solutions of a class of second-order nonlinear differential equations . . ........... 